\title{
Characterizations of Interval-Valued Intuitionistic Fuzzy n-Fold Positive Implicative Deal of BCK-Algebras
}

\author{
R. Durga Prasad ${ }^{1}$, B. Satyanarayana ${ }^{2}$ and G. Gebregziabiher ${ }^{3}$ \\ ${ }^{1,3}$ Department of Mathematics, College of Natural ana Coputationl Science, \\ Mizan-Tepi University,Ethiopia. \\ ${ }^{2}$ Department of Mathematics, Acharya Nagarjuna University, Nagarjuna Nagar-522510, \\ Andhra Pradesh,India. \\ ${ }^{1}$ E-mail: durgaprasad.fuzzy@gmail.com , ${ }^{2}$ E-mail: drbsn63@gmail.com , ${ }^{3}$ E-mail: gebremathlab@ gmail.com
}

\begin{abstract}
Using the concept of interval-valued intuitionistic fuzzy set, the notion of interval-valued intuitionistic fuzzy $\mathrm{n}$-fold BCK-ideals and interval-valued intuitionistic fuzzy n-fold positive implicative ideas are introduced, in BCK-Algebras and investigate some of its related properties. Characterizations of these notions and extension property of interval-valued intuitionistic fuzzy n-fold positive implicative ideal are investigated.
\end{abstract}

Keywords: Interval-valued intuitionistic fuzzy n-fold positive implicative ideal, Interval-valued intuitionistic fuzzy n-fold BCK-ideal.

2010 MSC No: 03E72, 06F35, 03G25.

\section{Introduction :}

For the general development of BCK-algebras, the ideal theory plays an important role. In [7] 1966 by K. Iseki and Y. Imai introduced a new notion called a BCK-algebras, and then many researchers have investigate various properties of this algebra. Hung and Chen [6] introduced the notion of $n$-fold implicative ideals, n-fold (weak) commutative ideals, n-fold positive implicative ideals and investigate some of its properties. Jun and Kim [9], introduced the notions of n-fold fuzzy positive implicative ideals in BCK-algebras and investigate some of its related properties. Satyanarayana and Durga Prasad [14], introduced the intuitionistic fuzzification of $n$-fold BCK-ideal and $\mathrm{n}$-fold positive implicative ideal in BCK-algebras and some of its related properties are investigated.

In this paper, we apply the concept of interval-valued intuitionistic fuzzy set to $\mathrm{n}$-fold BCKideals and n-fold positive implicative ideals in BCK-algebras and introduced the notion of intervalvalued intuitionistic fuzzy $\mathrm{n}$-fold BCK-ideal and interval-valued intuitionistic fuzzy n-fold positive implicative ideal and we gave relations between these notions and investigate some of its related properties. Characterizations of these notions and extension property of interval-valued intuitionistic fuzzy n-fold positive implicative ideals are investigated. 


\section{Preliminaries :}

Definition 2.1. Let $X$ be a set with a binary operation "*" and a constant " 0 ". Then $(X, *, 0)$ is called BCK-algebra, if it satisfies the following conditions.

$(\mathrm{BCK}-1)((\mathrm{x} * \mathrm{y}) *(\mathrm{x} * \mathrm{z})) *(\mathrm{z} * \mathrm{y})=0$,

$(\mathrm{BCK}-2)(\mathrm{x} *(\mathrm{x} * \mathrm{y})) * \mathrm{y}=0$,

$(\mathrm{BCK}-3) \quad \mathrm{x} * \mathrm{x}=0$

$(\mathrm{BCK}-4) 0 * \mathrm{x}=0$

$(B C K-5) \mathrm{x} * \mathrm{y}=0$ and $\mathrm{y} * \mathrm{x}=0$ imply $\mathrm{x}=\mathrm{y}$, for all $\mathrm{x}, \mathrm{y}, \mathrm{z} \in \mathrm{X}$

We can define a binary relation $\leq$ on $X$ by letting $\mathrm{x} \leq \mathrm{y}$ if and only if $\mathrm{x} * \mathrm{y}=0$. Then $(\mathrm{X}, \leq)$ is a partially ordered set with least element " 0 " and $(\mathrm{X}, *, 0)$ is a BCK-algebra if and only if, it satisfies the following: For all $\mathrm{x}, \mathrm{y}, \mathrm{z} \in \mathrm{X}$

(i) $((\mathrm{x} * \mathrm{y}) *(\mathrm{x} * \mathrm{z})) \leq(\mathrm{z} * \mathrm{y})$, (ii) $(\mathrm{x} *(\mathrm{x} * \mathrm{y})) \leq \mathrm{y}$, (iii) $\mathrm{x} \leq \mathrm{x}$, (iv) $0 \leq \mathrm{x}$

(v) $\mathrm{x} \leq \mathrm{y}$ and $\mathrm{y} \leq \mathrm{x}$ imply that $\mathrm{x}=\mathrm{y}$,

In a $\mathrm{BCK}$-algebra $(\mathrm{X}, *, 0)$, we have the following properties:

$(\mathrm{P} 1) \mathrm{x} * 0=\mathrm{x},(\mathrm{P} 2) \mathrm{x} * \mathrm{y} \leq \mathrm{x},(\mathrm{P} 3)(\mathrm{x} * \mathrm{y}) * \mathrm{z}=(\mathrm{x} * \mathrm{z}) * \mathrm{y},(\mathrm{P} 4)(\mathrm{x} * \mathrm{z}) *(\mathrm{y} * \mathrm{z}) \leq \mathrm{x} * \mathrm{y}$,

$(\mathrm{P} 5) \mathrm{x} *(\mathrm{x} *(\mathrm{x} * \mathrm{y}))=\mathrm{x} * \mathrm{y},(\mathrm{P} 6) \mathrm{x} \leq \mathrm{y} \Rightarrow \mathrm{x} * \mathrm{z} \leq \mathrm{y} * \mathrm{z}$ and $\mathrm{z} * \mathrm{y} \leq \mathrm{z} * \mathrm{x}$

$(\mathrm{P} 7) \mathrm{x} * \mathrm{y} \leq \mathrm{z}$ implies $\mathrm{x} * \mathrm{z} \leq \mathrm{y}$, for all $\mathrm{x}, \mathrm{y}, \mathrm{z} \in \mathrm{X}$.

Throughout this paper $X$ will always mean a BCK-algebra unless otherwise specified.

A non-empty sub-set $I$ of $X$ is said to be sub-algebra of $X$ if for $\mathrm{x}, \mathrm{y} \in \mathrm{I} \Rightarrow \mathrm{X} * \mathrm{y} \in \mathrm{I}$

A non-empty subset $I$ of $X$ is called an ideal of $X$ if $\left(I_{1}\right) 0 \in I\left(I_{2}\right) x * y$ and $y \in I \Rightarrow x \in I$ for every $\mathrm{x}, \mathrm{y} \in \mathrm{X}$, is said to be an $\mathrm{n}$-fold positive implicative ideal of $\mathrm{X}$ if $\left(\mathrm{I}_{1}\right)$ and $\left(\mathrm{I}_{3}\right)$ there exists a fixed $\mathrm{n} \in \mathrm{X}$ Such that $\quad(\mathrm{x} * \mathrm{y}) * \mathrm{z} \in$ and $\mathrm{y} * \mathrm{z}^{\mathrm{n}} \in \mathrm{I} \Rightarrow \mathrm{X} * \mathrm{z}^{\mathrm{n}} \in \mathrm{I}$ for every $\mathrm{x}, \mathrm{y}, \mathrm{z} \in \mathrm{X}$, is said to be an $\mathrm{n}$-fold BCK-ideal of $\mathrm{X}$ if $\left(\mathrm{I}_{1}\right)$ and $\left(\mathrm{I}_{3}\right)$ there exists a fixed $\mathrm{n} \in \mathrm{X}$ Such that $\left(\mathrm{x} * \mathrm{y}{ }^{\mathrm{n}+1}\right) * \mathrm{z} \in \mathrm{I}$ and $\mathrm{z} \in \mathrm{I} \Rightarrow \mathrm{x} * \mathrm{y}^{\mathrm{n}} \in \mathrm{I}$ for every $\mathrm{x}, \mathrm{y}, \mathrm{z} \in \mathrm{X}$. For any elements $\mathrm{x}$ and $\mathrm{y}$ of $\mathrm{X}, \mathrm{x} * \mathrm{y}{ }^{\mathrm{n}}$ denotes $(\ldots \ldots .((\mathrm{x} * \mathrm{y}) * \mathrm{y}) * \ldots \ldots .). * \mathrm{y}$ in which ' $\mathrm{y}$ ' occurs $\mathrm{n}$-times.

An interval-valued intuitionistic fuzzy set (i-v IFS, shortly) " $\tilde{\mathrm{A}}$ ” over $\mathrm{X}$ is an object having the form $\tilde{\mathrm{A}}=\left\{\left(\mathrm{x}, \tilde{\mu}_{\mathrm{A}}, \tilde{\lambda}_{\mathrm{A}}\right): \mathrm{x} \in \mathrm{X}\right\}$, where $\tilde{\mu}_{\mathrm{A}}(\mathrm{x}): \mathrm{X} \rightarrow \mathrm{D}[0,1]$ and $\tilde{\lambda}_{\mathrm{A}}(\mathrm{x}): \mathrm{X} \rightarrow \mathrm{D}[0,1]$, the intervals $\tilde{\mu}_{\mathrm{A}}(\mathrm{x})$ and $\tilde{\lambda}_{\mathrm{A}}(\mathrm{x})$ denotes the intervals of the degree of membership and the degree of the nonmembership of the element $\mathrm{x}$ to the set $\tilde{\mathrm{A}}$, where $\tilde{\mu}_{\mathrm{A}}(\mathrm{x})=\left[\mu_{\mathrm{A}}^{-}(\mathrm{x}), \mu_{\mathrm{A}}^{+}(\mathrm{x})\right]$ and $\tilde{\lambda}_{\mathrm{A}}(\mathrm{x})=\left[\lambda_{\mathrm{A}}^{-}(\mathrm{x}), \lambda_{\mathrm{A}}^{+}(\mathrm{x})\right]$ for all $\mathrm{x} \in \mathrm{X}$ with the condition $[0,0] \leq \tilde{\mu}_{\mathrm{A}}(\mathrm{x})+\tilde{\lambda}_{\mathrm{A}}(\mathrm{x}) \leq[1,1]$ for all $\mathrm{x} \in \mathrm{X}$ . For the sake of simplicity, we use the symbol $\tilde{\mathrm{A}}=\left(\tilde{\mu}_{\mathrm{A}}, \tilde{\lambda}_{\mathrm{A}}\right)$.

Definition. 2.2. An i-v IFS $\widetilde{A}=\left(X, \tilde{\mu}_{A}, \tilde{\lambda}_{A}\right)$ in $X$ is an interval-valued intuitionistic fuzzy ideal of $X$, if it satisfies

(i-v IF1) $\tilde{\mu}_{\mathrm{A}}(0) \geq \tilde{\mu}_{\mathrm{A}}(\mathrm{x})$ and $\tilde{\lambda}_{\mathrm{A}}(0) \leq \tilde{\lambda}_{\mathrm{A}}(\mathrm{x})$

(i-v IF2) $\tilde{\mu}_{\mathrm{A}}(\mathrm{x}) \geq \min \left\{\tilde{\mu}_{\mathrm{A}}(\mathrm{x} * \mathrm{y}), \tilde{\mu}_{\mathrm{A}}(\mathrm{x})\right\}$

(i-v IF3) $\tilde{\lambda}_{\mathrm{A}}(\mathrm{x}) \leq \min \left\{\tilde{\lambda}_{\mathrm{A}}(\mathrm{x} * \mathrm{y}), \tilde{\lambda}_{\mathrm{A}}(\mathrm{y})\right\}$ for all $\mathrm{x}, \mathrm{y} \in \mathrm{X}$.

Theorem.2.3. An intuitionistic fuzzy sub-algebra $A=\left(X, \mu_{A}, \lambda_{A}\right)$ is an intuitionistic fuzzy ideal of $X$ if and only if for $\mathrm{x}, \mathrm{y}, \mathrm{z} \in \mathrm{X}, \mathrm{x} * \mathrm{y} \leq \mathrm{z} \Rightarrow \mu_{\mathrm{A}}(\mathrm{x}) \geq \min \left\{\mu_{\mathrm{A}}(\mathrm{y}), \mu_{\mathrm{A}}(\mathrm{z})\right\}$ and $\lambda_{\mathrm{A}}(\mathrm{x}) \leq \max \left\{\lambda_{\mathrm{A}}(\mathrm{y}), \lambda_{\mathrm{A}}(\mathrm{z})\right\}$.

Theorem 2.4. Let $\tilde{\mathrm{A}}=\left(\mathrm{X}, \tilde{\mu}_{\mathrm{A}}, \tilde{\lambda}_{\mathrm{A}}\right)$ be an interval valued intuitionistic fuzzy ideal of $\mathrm{X}$.

If $\mathrm{x} \leq \mathrm{y}$ in $\mathrm{X}$, then $\tilde{\mu}_{\mathrm{A}}(\mathrm{x}) \geq \tilde{\mu}_{\mathrm{A}}(\mathrm{y}), \tilde{\lambda}_{\mathrm{A}}(\mathrm{x}) \leq \tilde{\lambda}_{\mathrm{A}}(\mathrm{y})$, that is, $\tilde{\mu}_{\mathrm{A}}$ is order-reversing and $\tilde{\lambda}_{\mathrm{A}}$ is orderpreserving. 


\section{Interval-valued intuitionistic fuzzy n-fold BCK-ideals of BCK-algebras :}

Definition 3.1. An i-v IFS $\tilde{A}=\left(\tilde{\mu}_{A}, \tilde{\lambda}_{A}\right)$ in $X$ is an interval-valued intuitionistic fuzzy n-fold BCKideal of $X$, if it satisfies

$$
\begin{aligned}
& \left(\mathrm{i}-\mathrm{vBCKI}{ }^{\mathrm{n}} 1\right) \tilde{\mu}_{\mathrm{A}}(0) \geq \tilde{\mu}_{\mathrm{A}}(\mathrm{x}), \tilde{\lambda}_{\mathrm{A}}(0) \leq \tilde{\lambda}_{\mathrm{A}}(\mathrm{x}) \text { and there exists a fixed } \mathrm{n} \in \mathrm{N} \text { such that } \\
& \left(\mathrm{i}-\mathrm{vBCKI} \mathrm{I}^{\mathrm{n}} 2\right) \tilde{\mu}_{\mathrm{A}}\left(\mathrm{x} * \mathrm{y}{ }^{\mathrm{n}}\right) \geq \min \left\{\tilde{\mu}_{\mathrm{A}}\left(\left(\mathrm{x} * \mathrm{y}^{\mathrm{n}+1}\right) * \mathrm{z}\right), \tilde{\mu}_{\mathrm{A}}(\mathrm{z})\right\} \\
& \left(\mathrm{i}-\mathrm{vBCKI}{ }^{\mathrm{n}} 3\right) \tilde{\lambda}_{\mathrm{A}}\left(\mathrm{x} * \mathrm{y}{ }^{\mathrm{n}}\right) \leq \max \left\{\tilde{\lambda}_{\mathrm{A}}\left(\left(\mathrm{x} * \mathrm{y}^{\mathrm{n}+1}\right) * \mathrm{z}\right), \tilde{\lambda}_{A}(\mathrm{z})\right\} \text { for all } \mathrm{x}, \mathrm{y}, \mathrm{z} \in \mathrm{X} .
\end{aligned}
$$

\section{Theorem 3.2}

Every interval-valued intuitionistic fuzzy $\mathrm{n}$-fold BCK- ideal of $\mathrm{X}$ is an interval-valued intuitionistic fuzzy deal of $X$.

\section{Proof:}

Put $\mathrm{y}=0$ in $\left(\mathrm{i}-\mathrm{vBCKI}^{\mathrm{n}}{ }_{2}\right)$ and $\left(\mathrm{i}-\mathrm{vBCKI}^{\mathrm{n}}{ }^{3}\right)$ we get the proof of the result.

The following example shows that the converse of theorem 3.2 may not be true.

\section{Example 3.3}

Let $X=N \cup\{0\}$, where $N$ is the set of natural numbers, in which the operation" $*$ " is defined by $\mathrm{x} * \mathrm{y}=\max \{0, \mathrm{x}-\mathrm{y}\}$ for all $\mathrm{x}, \mathrm{y} \in \mathrm{X}$. Then $\mathrm{X}$ is a BCK-algebra.

Let $\tilde{\mathrm{A}}=\left(\tilde{\mu}_{\mathrm{A}}, \tilde{\lambda}_{\mathrm{A}}\right)$ be an $\mathrm{i}-\mathrm{v}$ IFS in $\mathrm{X}$ given by

$$
\tilde{\mu}_{\mathrm{A}}(0)=[0.7,0.8]>[0.3,0.4]=\tilde{\mu}_{\mathrm{A}}(\mathrm{x}) \text { and } \tilde{\lambda}_{\mathrm{A}}(0)=[0.1,0.2]<[0.3,0.4]=\tilde{\lambda}_{\mathrm{A}}(\mathrm{x}),
$$

for all $\mathrm{x}(\neq 0) \in \mathrm{X}$. Then $\tilde{\mathrm{A}}$ is an interval-valued intuitionistic fuzzy ideal of $\mathrm{X}$ but it is not an intervalvalued intuitionistic fuzzy 2-fold BCK-ideal of $X$, because $\left.\tilde{\mu}_{\mathrm{A}}\left(5 * 2^{2}\right)=\tilde{\mu}_{\mathrm{A}}(1)=[0.3,0.4]<[0.7,0.8]=\tilde{\mu}_{\mathrm{A}}(0)=\min \left\{\tilde{\mu}_{\mathrm{A}}\left(\left(5 * 2^{3}\right)\right) * 0\right), \tilde{\mu}_{\mathrm{A}}(0)\right\}$ and $\left.\lambda_{\mathrm{A}}\left(5 * 2^{2}\right)=\tilde{\lambda}_{\mathrm{A}}(1)=[0.4,0.45]>[0.1,0.2]=\tilde{\lambda}_{\mathrm{A}}(0)=\max \left\{\tilde{\lambda}_{\mathrm{A}}\left(\left(5 * 2^{3}\right)\right) * 0\right), \tilde{\lambda}_{\mathrm{A}}(0)\right\}$

We give a condition for an interval-valued intuitionistic fuzzy ideal to be an intuitionistic fuzzy $n$-fold BCK-ideal

\section{Proposition 3.4}

Let $\tilde{\mathrm{A}}=\left(\tilde{\mu}_{\mathrm{A}}, \tilde{\lambda}_{\mathrm{A}}\right)$ be an interval-valued intuitionistic fuzzy ideal of $\mathrm{X}$. Then $\tilde{\mathrm{A}}$ is an interval-valued intuitionistic fuzzy $\mathrm{n}$-fold $\mathrm{BCK}$-ideal of $\mathrm{X}$ if and only if it satisfies the following inequalities $\tilde{\mu}_{A}\left(x * y^{n}\right) \geq \tilde{\mu}_{A}\left(x * y^{n+1}\right)$ and $\tilde{\lambda}_{A}\left(x * y^{n}\right) \leq \tilde{\lambda}_{A}\left(x * y^{n+1}\right)$ for all $x, y \in X$.

\section{Proof:}

Put $\mathrm{z}=0$ in (i-v BCKI ${ }^{\mathrm{n}}$ 2) and (i-v BCKI $\left.{ }^{\mathrm{n}} 3\right)$, we get

$$
\tilde{\mu}_{\mathrm{A}}\left(\mathrm{x} * \mathrm{y}^{\mathrm{n}}\right) \geq \min \left\{\tilde{\mu}_{\mathrm{A}}\left(\left(\mathrm{x} * \mathrm{y}^{\mathrm{n}+1}\right) * 0\right), \tilde{\mu}_{\mathrm{A}}(0)\right\}=\min \left\{\tilde{\mu}_{\mathrm{A}}\left(\mathrm{x} * \mathrm{y}^{\mathrm{n}+1}\right), \tilde{\mu}_{\mathrm{A}}(0)\right\}=\tilde{\mu}_{\mathrm{A}}\left(\mathrm{x} * \mathrm{y}^{\mathrm{n}+1}\right\}
$$

and $\tilde{\lambda}_{A}\left(\mathrm{x} * \mathrm{y}^{\mathrm{n}}\right) \leq \max \left\{\tilde{\lambda}_{\mathrm{A}}\left(\left(\mathrm{x} * \mathrm{y}^{\mathrm{n}+1}\right) * 0\right), \tilde{\lambda}_{\mathrm{A}}(0\}=\max \left\{\tilde{\lambda}_{\mathrm{A}}\left(\mathrm{x} * \mathrm{y}^{\mathrm{n}+1}\right), \tilde{\lambda}_{\mathrm{A}}(0)\right\}=\tilde{\lambda}_{\mathrm{A}}\left(\mathrm{x} * \mathrm{y}^{\mathrm{n}+1}\right\}\right.$

Therefore, $\tilde{\mu}_{A}\left(x * y^{n}\right) \geq \tilde{\mu}_{A}\left(x * y^{n+1}\right)$ and $\tilde{\lambda}_{A}\left(x * y^{n}\right) \leq \tilde{\lambda}_{A}\left(x * y^{n+1}\right)$ for all $x, y \in X$.

Conversely, Since (i-vIF2) and (i-vIF3) we get

$$
\tilde{\mu}_{\mathrm{A}}\left(\mathrm{x} * \mathrm{y}^{\mathrm{n}}\right) \geq \tilde{\mu}_{\mathrm{A}}\left(\mathrm{x} * \mathrm{y}^{\mathrm{n}+1}\right) \geq \min \left\{\tilde{\mu}_{\mathrm{A}}\left(\left(\mathrm{x} * \mathrm{y}^{\mathrm{n}+1}\right) * \mathrm{z}\right), \tilde{\mu}_{\mathrm{A}}(\mathrm{z})\right\} \text { and }
$$




$$
\tilde{\lambda}_{\mathrm{A}}\left(\mathrm{x} * \mathrm{y}^{\mathrm{n}}\right) \leq \tilde{\lambda}_{\mathrm{A}}\left(\mathrm{x} * \mathrm{y}^{\mathrm{n}+1}\right) \leq \max \left\{\tilde{\lambda}_{\mathrm{A}}\left(\left(\mathrm{x} * \mathrm{y}^{\mathrm{n}+1}\right) * \mathrm{z}\right), \tilde{\lambda}_{\mathrm{A}}(\mathrm{z})\right\} \text { for all } \mathrm{x}, \mathrm{y}, \mathrm{z} \in \mathrm{X} .
$$

Thus $\tilde{\mathrm{A}}=\left(\tilde{\mu}_{\mathrm{A}}, \tilde{\lambda}_{\mathrm{A}}\right)$ is an interval-valued intuitionistic fuzzy $\mathrm{n}$-fold BCK-ideal of $\mathrm{X}$

Corollary 3. 5

Every interval-valued intuitionistic fuzzy n-fold BCK-ideal

$\tilde{\mathrm{A}}=\left(\tilde{\mu}_{\mathrm{A}}, \tilde{\lambda}_{\mathrm{A}}\right)$ of $\mathrm{X}$ satisfies the inequalities $\tilde{\mu}_{\mathrm{A}}\left(\mathrm{x} * \mathrm{y}^{\mathrm{n}}\right) \geq \tilde{\mu}_{\mathrm{A}}\left(\mathrm{x} * \mathrm{y}^{\mathrm{n}+\mathrm{k}}\right)$ and

$\tilde{\lambda}_{A}\left(x * y^{n}\right) \leq \tilde{\lambda}_{A}\left(x * y^{n+k}\right)$ for all $x, y \in X$ and $k \in N$

Proof:

Using the proposition 3.4, the proof is straightforward by Induction

\section{Theorem 3.6.}

An interval-valued intuitionistic fuzzy set $\tilde{A}=\left(\tilde{\mu}_{A}, \tilde{\lambda}_{A}\right)$ in $X$ is an interval-valued intuitionistic fuzzy $\mathrm{n}$-fold BCK-ideal of $X$ if and only if the non-empty upper $\tilde{s}$-level cut $U\left(\tilde{\mu}_{A} ; \tilde{s}\right)$ and the non-empty lower $\tilde{\mathrm{t}}$-level cut $\mathrm{L}\left(\tilde{\lambda}_{A} ; \tilde{\mathrm{t}}\right)$ are $\mathrm{n}$-fold BCK-ideals of $X$ for any $\tilde{s}, \tilde{\mathrm{t}} \in \mathrm{D}[0,1]$.

Proof: The proof is straight forward.

\section{Interval-valued intuitionistic fuzzy n-fold positive implicative ideals of BCK-algebras :}

\section{Definition 4.1}

An i-v IFS $\tilde{\mathrm{A}}=\left(\tilde{\mu}_{\mathrm{A}}, \tilde{\lambda}_{\mathrm{A}}\right)$ in $\mathrm{X}$ is an interval-valued intuitionistic fuzzy n-fold positive implicative ideal (i-vIFPI ${ }^{\mathrm{n}}$-ideal ) of $\mathrm{X}$ if it satisfies

$\left(\mathrm{i}-\mathrm{vIFPI}^{\mathrm{n}} 1\right) \tilde{\mu}_{\mathrm{A}}(0) \geq \tilde{\mu}_{\mathrm{A}}(\mathrm{x}), \tilde{\lambda}_{\mathrm{A}}(0) \leq \tilde{\lambda}_{\mathrm{A}}(\mathrm{x})$ and there exists a fixed $\mathrm{n} \in \mathrm{N}$ such that

$\left(\mathrm{i}-\mathrm{vIFPI}{ }^{\mathrm{n}} 2\right) \tilde{\mu}_{\mathrm{A}}\left(\mathrm{x} * \mathrm{z}^{\mathrm{n}}\right) \geq \min \left\{\tilde{\mu}_{\mathrm{A}}\left((\mathrm{x} * \mathrm{y}) * \mathrm{z}^{\mathrm{n}}\right), \tilde{\mu}_{\mathrm{A}}\left(\mathrm{y} * \mathrm{z}^{\mathrm{n}}\right)\right\}$

$\left(\mathrm{i}-\mathrm{vIFPI}^{\mathrm{n}} 3\right) \tilde{\lambda}_{\mathrm{A}}\left(\mathrm{x} * \mathrm{z}^{\mathrm{n}}\right) \leq \max \left\{\tilde{\lambda}_{A}\left((\mathrm{x} * \mathrm{y}) * \mathrm{z}^{\mathrm{n}}\right), \tilde{\lambda}_{A}\left(\mathrm{y} * \mathrm{z}^{\mathrm{n}}\right)\right\}$ for all $\mathrm{x}, \mathrm{y}, \mathrm{z} \in \mathrm{X}$.

Example 4.2

Let $X=\{0,1,2\}$ be a BCK-algebra with the following Cayley table

\begin{tabular}{|l|l|l|l|}
\hline$*$ & 0 & 1 & 2 \\
\hline 0 & 0 & 0 & 0 \\
\hline 1 & 1 & 0 & 0 \\
\hline 2 & 2 & 2 & 0 \\
\hline
\end{tabular}

Define an i-v IFS $\tilde{\mathrm{A}}=\left(\tilde{\mu}_{\mathrm{A}}, \tilde{\lambda}_{\mathrm{A}}\right)$ in $\mathrm{X}$ by

$$
\begin{aligned}
& \tilde{\mu}_{\mathrm{A}}(0)=[0.75,0.8], \tilde{\mu}_{\mathrm{A}}(1)=[0.6,0.7], \tilde{\mu}_{\mathrm{A}}(2)=[0.2,0.3] \text { and } \\
& \tilde{\lambda}_{\mathrm{A}}(0)=[0.1,0.15], \tilde{\lambda}_{\mathrm{A}}(1)=[0.2,0.25], \tilde{\mu}_{\mathrm{A}}(2)=[0.4,0.45]
\end{aligned}
$$

Then $\tilde{A}=\left(\tilde{\mu}_{A}, \tilde{\lambda}_{A}\right)$ is an interval-valued intuitionist fuzzy $n$-fold positive implicative ideal of $\mathrm{X}$ for all $\mathrm{n} \in \mathrm{N}$ 
Characterizations of Interval-valued intuitionistic fuzzy n-fold positive implicative deal of BCK-algebras

\section{Theorem 4.3}

Every interval-valued intuitionistic fuzzy n-fold positive implicative ideal X must be interval-valued intuitionistic fuzzy idea of $\mathrm{X}$.

\section{Proof:}

Put $\mathrm{z}=0$ in (i-vIFPI ${ }^{\mathrm{n}}$ ) and (i-vIFPI $\left.{ }^{\mathrm{n}} 3\right)$, we get the proof of the result.

The following example shows that the converse of the Theorem 4.3 not true in general.

\section{Example 4.4}

Let $\mathrm{X}=\mathrm{N} \cup\{0\}$, where $\mathrm{N}$ is the set of natural numbers, in which the operation $*$ is defined by $\mathrm{x} * \mathrm{y}=\max \{0, \mathrm{x}-\mathrm{y}\}$ for all $\mathrm{x}, \mathrm{y} \in \mathrm{X}$. Then $\mathrm{X}$ is a BCK-algebra.

Let $\tilde{\mathrm{A}}=\left(\tilde{\mu}_{\mathrm{A}}, \tilde{\lambda}_{\mathrm{A}}\right)$ be an i-v IFS in $\mathrm{X}$ given by

$$
\tilde{\mu}_{\mathrm{A}}(0)=[0.7,0.8]>[0.3,0.4]=\tilde{\mu}_{\mathrm{A}}(\mathrm{x}) \text { and } \tilde{\lambda}_{\mathrm{A}}(0)=[0.1,0.2]<[0.4,0.45]=\tilde{\lambda}_{\mathrm{A}}(\mathrm{x}) \text {, }
$$

for all $\mathrm{x}(\neq 0) \in \mathrm{X}$. Then $\tilde{A}$ is an interval-valued intuitionistic fuzzy ideal of $X$ but $\tilde{A}$ is not an intervalvalued intuitionistic fuzzy 2 -fold Positive implicative-ideal of $X$, because

$$
\begin{aligned}
\mu_{\mathrm{A}}\left(13 * 5^{2}\right) & =\mu_{\mathrm{A}}(3)=[0.3,0.4]<[0.7,0.8]=\tilde{\mu}_{\mathrm{A}}(0) \\
& \left.=\min \left\{\tilde{\mu}_{\mathrm{A}}\left((13 * 3) * 5^{2}\right)\right), \tilde{\mu}_{\mathrm{A}}\left(3 * 5^{2}\right)\right\} \\
\tilde{\lambda}_{\mathrm{A}}\left(13 * 5^{2}\right) & =\tilde{\lambda}_{\mathrm{A}}(3)=[0.4,0.45]>[0.1,0.2]=\lambda_{\mathrm{A}}(0) \\
& \left.=\max \left\{\lambda_{\mathrm{A}}\left((13 * 3) * 5^{2}\right)\right), \lambda_{\mathrm{A}}\left(3 * 5^{2}\right)\right\}
\end{aligned}
$$

\section{Theorem 4.5}

Let $\tilde{\mathrm{A}}=\left(\tilde{\mu}_{\mathrm{A}}, \tilde{\lambda}_{\mathrm{A}}\right)$ be an interval-valued intuitionistic fuzzy ideal of $\mathrm{X}$. Then $\tilde{\mathrm{A}}$ is an interval-valued intuitionistic fuzzy $\mathrm{n}$-fold positive implicative ideal of $\mathrm{X}$ if and only if it satisfies the inequalities $\tilde{\mu}_{A}\left(\left(\mathrm{x} * \mathrm{z}^{\mathrm{n}}\right) *\left(\mathrm{y} * \mathrm{z}^{\mathrm{n}}\right)\right) \geq \tilde{\mu}_{\mathrm{A}}\left((\mathrm{x} * \mathrm{y}) * \mathrm{z}^{\mathrm{n}}\right)$ and $\tilde{\lambda}_{\mathrm{A}}\left(\left(\mathrm{x} * \mathrm{z}^{\mathrm{n}}\right) *\left(\mathrm{y} * \mathrm{z}^{\mathrm{n}}\right)\right) \leq \tilde{\lambda}_{\mathrm{A}}\left((\mathrm{x} * \mathrm{y}) * \mathrm{z}^{\mathrm{n}}\right)$ for all $\mathrm{x}, \mathrm{y}, \mathrm{z} \in \mathrm{X}$. Proof:

Let $\mathrm{x}, \mathrm{y}, \mathrm{z} \in \mathrm{X}$ and $\mathrm{a}=\mathrm{x} *\left(\mathrm{y} * \mathrm{z}^{\mathrm{n}}\right)$ and $\mathrm{b}=\mathrm{x} * \mathrm{y}$. Since, for all $\left.\mathrm{x}, \mathrm{y}, \mathrm{z} \in \mathrm{X},\left(\left(\mathrm{x} *\left(\mathrm{y} * \mathrm{z}{ }^{\mathrm{n}}\right)\right) *(\mathrm{x} * \mathrm{y})\right) * \mathrm{z}{ }^{\mathrm{n}}\right) \leq\left(\mathrm{y} *\left(\mathrm{y} * \mathrm{z}^{\mathrm{n}}\right)\right) * \mathrm{z}^{\mathrm{n}}$, By BCK-1 and Theorem 2.4, we have $\left.\tilde{\mu}_{\mathrm{A}}\left(\left(\left(\mathrm{x} *\left(\mathrm{y} * \mathrm{z}^{\mathrm{n}}\right)\right) *(\mathrm{x} * \mathrm{y})\right) * \mathrm{z}^{\mathrm{n}}\right)\right) \geq \tilde{\mu}_{\mathrm{A}}\left(\left(\mathrm{y} *\left(\mathrm{y} * \mathrm{z}^{\mathrm{n}}\right)\right) * \mathrm{z}^{\mathrm{n}}\right)$ and

$$
\left.\tilde{\lambda}_{\mathrm{A}}\left(\left(\left(\mathrm{x} *\left(\mathrm{y} * \mathrm{z}^{\mathrm{n}}\right)\right) *(\mathrm{x} * \mathrm{y})\right) * \mathrm{z}^{\mathrm{n}}\right)\right) \leq \tilde{\lambda}_{\mathrm{A}}\left(\left(\mathrm{y} *\left(\mathrm{y} * \mathrm{z}^{\mathrm{n}}\right)\right) * \mathrm{z}^{\mathrm{n}}\right)
$$

Then $\tilde{\mu}_{\mathrm{A}}\left((\mathrm{a} * \mathrm{~b}) * \mathrm{z}^{\mathrm{n}}\right)=\tilde{\mu}_{\mathrm{A}}\left(\left(\left(\mathrm{x} *\left(\mathrm{y} * \mathrm{z}^{\mathrm{n}}\right)\right) *(\mathrm{x} * \mathrm{y})\right) * \mathrm{z}^{\mathrm{n}}\right) \geq \tilde{\mu}_{\mathrm{A}}\left(\left(\mathrm{y} *\left(\mathrm{y} * \mathrm{z}^{\mathrm{n}}\right)\right) * \mathrm{z}^{\mathrm{n}}\right)$

$$
\begin{aligned}
& =\tilde{\mu}_{\mathrm{A}}\left(\left(\mathrm{y} * \mathrm{z}^{\mathrm{n}}\right) *\left(\mathrm{y} * \mathrm{z}^{\mathrm{n}}\right)\right) \quad[\mathrm{By} \mathrm{P} 3] \\
& =\tilde{\mu}_{A}(0) \text { [By BCK-3] }
\end{aligned}
$$

and so $\tilde{\mu}_{A}\left((a * b) * z^{n}\right)=\tilde{\mu}_{A}(0)$.

And $\left.\tilde{\lambda}_{\mathrm{A}}\left((\mathrm{a} * \mathrm{~b}) * \mathrm{z}^{\mathrm{n}}\right)=\tilde{\lambda}_{\mathrm{A}}\left(\left(\left(\mathrm{x} *\left(\mathrm{y} * \mathrm{z}^{\mathrm{n}}\right)\right) *(\mathrm{x} * \mathrm{y})\right) * \mathrm{z}^{\mathrm{n}}\right) \leq \tilde{\lambda}_{\mathrm{A}}\left(\left(\mathrm{y} *\left(\mathrm{y} * \mathrm{z}^{\mathrm{n}}\right)\right) * \mathrm{z}^{\mathrm{n}}\right)\right)$

$$
\begin{aligned}
& =\tilde{\lambda}_{\mathrm{A}}\left(\left(\mathrm{y} * \mathrm{z}^{\mathrm{n}}\right) *\left(\mathrm{y} * \mathrm{z}^{\mathrm{n}}\right)\right) \quad \text { [By P3] } \\
& =\tilde{\lambda}_{\mathrm{A}}(0) \text { [By BCK-3] }
\end{aligned}
$$


and so $\tilde{\lambda}_{A}\left((a * b) * z^{n}\right)=\tilde{\lambda}_{A}(0)$.

Using (P3), (i-vIFPI ${ }^{\mathrm{n}}$ 2) and (i-vIFPI ${ }^{\mathrm{n}}$ 3) we obtain

$$
\begin{aligned}
\tilde{\mu}_{\mathrm{A}}\left(\left(\mathrm{x} * \mathrm{z}^{\mathrm{n}}\right) *\left(\mathrm{y} * \mathrm{z}^{\mathrm{n}}\right)\right) & =\tilde{\mu}_{\mathrm{A}}\left(\left(\mathrm{x} *\left(\mathrm{y} * \mathrm{z}^{\mathrm{n}}\right)\right) * \mathrm{z}^{\mathrm{n}}\right)=\tilde{\mu}_{\mathrm{A}}\left(\mathrm{a} * \mathrm{z}^{\mathrm{n}}\right) \\
& \geq \min \left\{\tilde{\mu}_{\mathrm{A}}\left((\mathrm{a} * \mathrm{~b}) * \mathrm{z}^{\mathrm{n}}\right), \tilde{\mu}_{\mathrm{A}}\left(\mathrm{b} * \mathrm{z}^{\mathrm{n}}\right)\right\} \\
& =\min \left\{\tilde{\mu}_{\mathrm{A}}(0), \tilde{\mu}_{\mathrm{A}}\left(\mathrm{b} * \mathrm{z}^{\mathrm{n}}\right)\right\} \\
& =\tilde{\mu}_{\mathrm{A}}\left(\mathrm{b} * \mathrm{z}^{\mathrm{n}}\right)=\tilde{\mu}_{\mathrm{A}}\left((\mathrm{x} * \mathrm{y}) * \mathrm{z}^{\mathrm{n}}\right) \text { and } \\
\tilde{\lambda}_{\mathrm{A}}\left(\left(\mathrm{x} * \mathrm{z}^{\mathrm{n}}\right) *\left(\mathrm{y} * \mathrm{z}^{\mathrm{n}}\right)\right) & =\tilde{\lambda}_{\mathrm{A}}\left(\left(\mathrm{x} *\left(\mathrm{y} * \mathrm{z}^{\mathrm{n}}\right)\right) * \mathrm{z}^{\mathrm{n}}\right)=\tilde{\lambda}_{\mathrm{A}}\left(\mathrm{a} * \mathrm{z}^{\mathrm{n}}\right) \\
& \left.\leq \max _{\hat{\lambda}} \tilde{\lambda}_{\mathrm{A}}\left((\mathrm{a} * \mathrm{~b}) * \mathrm{z}^{\mathrm{n}}\right), \tilde{\lambda}_{\mathrm{A}}\left(\mathrm{b} * \mathrm{z}^{\mathrm{n}}\right)\right\} \\
& =\max _{\mathrm{max}}\left(\tilde{\lambda}_{\mathrm{A}}(0), \tilde{\lambda}_{\mathrm{A}}\left(\mathrm{b} * \mathrm{z}^{\mathrm{n}}\right)\right\} \\
& =\tilde{\lambda}_{\mathrm{A}}\left(\mathrm{b} * \mathrm{z}^{\mathrm{n}}\right)=\tilde{\lambda}_{\mathrm{A}}\left((\mathrm{x} * \mathrm{y}) * \mathrm{z}^{\mathrm{n}}\right)
\end{aligned}
$$

Thus $\tilde{\mu}_{\mathrm{A}}\left(\left(\mathrm{x} * \mathrm{z}^{\mathrm{n}}\right) *\left(\mathrm{y} * \mathrm{z}^{\mathrm{n}}\right)\right) \geq \tilde{\mu}_{\mathrm{A}}\left((\mathrm{x} * \mathrm{y}) * \mathrm{z}^{\mathrm{n}}\right)$ and

$\tilde{\lambda}_{\mathrm{A}}\left(\left(\mathrm{x} * \mathrm{z}^{\mathrm{n}}\right) *\left(\mathrm{y} * \mathrm{z}^{\mathrm{n}}\right)\right) \leq \tilde{\lambda}_{\mathrm{A}}\left((\mathrm{x} * \mathrm{y}) * \mathrm{z}^{\mathrm{n}}\right)$ for all $\mathrm{x}, \mathrm{y} \in \mathrm{X}$.

Covertly, For any $x, y, z \in X$. Using (i-vIF-2) and (i-vIF-3), we obtain

$$
\begin{aligned}
& \tilde{\mu}_{\mathrm{A}}\left(\mathrm{x} * \mathrm{z}^{\mathrm{n}}\right) \geq \min \left\{\tilde{\mu}_{\mathrm{A}}\left(\left(\mathrm{x} * \mathrm{z}^{\mathrm{n}}\right) *\left(\mathrm{y} * \mathrm{z}^{\mathrm{n}}\right)\right), \tilde{\mu}_{\mathrm{A}}\left(\mathrm{y} * \mathrm{z}^{\mathrm{n}}\right)\right\} \geq \min \left\{\tilde{\mu}_{\mathrm{A}}\left((\mathrm{x} * \mathrm{y}) * \mathrm{z}^{\mathrm{n}}\right), \tilde{\mu}_{\mathrm{A}}\left(\mathrm{y} * \mathrm{z}^{\mathrm{n}}\right)\right\} \text { and } \\
& \tilde{\lambda}_{\mathrm{A}}\left(\mathrm{x} * \mathrm{z}^{\mathrm{n}}\right) \leq \max \left\{\tilde{\lambda}_{\mathrm{A}}\left(\left(\mathrm{x} * \mathrm{z}^{\mathrm{n}}\right) *\left(\mathrm{y} * \mathrm{z}^{\mathrm{n}}\right)\right), \tilde{\lambda}_{\mathrm{A}}\left(\mathrm{y} * \mathrm{z}^{\mathrm{n}}\right)\right\} \leq \max \left\{\tilde{\lambda}_{\mathrm{A}}\left((\mathrm{x} * \mathrm{y}) * \mathrm{z}^{\mathrm{n}}\right), \tilde{\lambda}_{\mathrm{A}}\left(\mathrm{y} * \mathrm{z}^{\mathrm{n}}\right)\right\} \text { for all }
\end{aligned}
$$

$\mathrm{x}, \mathrm{y}, \mathrm{z} \in \mathrm{X}$. Thus $\tilde{\mathrm{A}}=\left(\tilde{\mu}_{\mathrm{A}}, \tilde{\lambda}_{\mathrm{A}}\right)$ is an interval-valued intuitionist fuzzy $\mathrm{n}$-fold positive implicative ideal of $\mathrm{X}$.

\section{Proposition 4.6}

Let $\tilde{A}=\left(\tilde{\mu}_{A}, \tilde{\lambda}_{A}\right)$ be an interval-valued intuitionistic fuzzy ideal of $X$. Then $\tilde{A}$ is an interval-valued intuitionistic fuzzy $n$-fold positive implicative ideal of $X$ then it satisfies the inequalities $\tilde{\mu}_{A}\left(x * y^{n}\right) \geq \tilde{\mu}_{A}\left((x * y) * y^{n}\right)$ and $\tilde{\lambda}_{A}\left(x * y^{n}\right) \leq \tilde{\lambda}_{A}\left((x * y) * y^{n}\right)$ for all $x, y \in X$.

\section{Proof:}

Put $\mathrm{z}=\mathrm{y}$ in (i-vIFPI ${ }^{\mathrm{n}}$ ) ) and (i-vIFPI $\left.{ }^{\mathrm{n}} 3\right)$, we get the proof of the result.

\section{Proposition 4.7}

Let $\tilde{\mathrm{A}}=\left(\tilde{\mu}_{\mathrm{A}}, \tilde{\lambda}_{\mathrm{A}}\right)$ be an interval-valued intuitionist fuzzy set of $\mathrm{X}$. Then $\tilde{\mathrm{A}}$ is an interval-valued intuitionistic fuzzy $n$-fold positive implicative ideal of $X$ if and only if it is an intuitionistic fuzzy $n$-fold BCK-ideal of $X$.

\section{Proof:}

Putting $\mathrm{z}=\mathrm{y}$ in (i-vIFPI ${ }^{\mathrm{n}}$ 2) and $\left(\mathrm{i}-\mathrm{vIFPI}^{\mathrm{n}} 3\right)$, we get

$$
\begin{aligned}
\tilde{\mu}_{A}\left(x * y^{n}\right) \geq \min \left\{\tilde{\mu}_{A}\left((x * y) * y^{n}\right), \tilde{\mu}_{A}\left(y * y^{n}\right)\right. & \left.=\min \left\{\tilde{\mu}_{A}\left(x * y^{n+1}\right), \tilde{\mu}_{A}(0)\right\}\right\} \\
& =\tilde{\mu}_{A}(x * y=1) \text { and }
\end{aligned}
$$




$$
\begin{aligned}
\tilde{\lambda}_{\mathrm{A}}\left(\mathrm{x} * \mathrm{y}^{\mathrm{n}}\right) \leq \max \left\{\tilde{\lambda}_{\mathrm{A}}\left((\mathrm{x} * \mathrm{y}) * \mathrm{y}^{\mathrm{n}}\right), \tilde{\lambda}_{\mathrm{A}}\left(\mathrm{y} * \mathrm{y}^{\mathrm{n}}\right)\right. & \left.=\max \left\{\tilde{\mu}_{\mathrm{A}}\left(\mathrm{x} * \mathrm{y}^{\mathrm{n}+1}\right), \tilde{\lambda}_{\mathrm{A}}(0)\right\}\right\} \\
& =\tilde{\lambda}_{\mathrm{A}}\left(\mathrm{x} * \mathrm{y}{ }^{\mathrm{n}+1}\right) .
\end{aligned}
$$

Therefore, $\tilde{\mu}_{A}\left(x * y^{n}\right) \geq \tilde{\mu}_{A}\left(x * y^{n+1}\right)$ and $\tilde{\lambda}_{A}\left(x * y^{n}\right) \leq \tilde{\lambda}_{A}\left(x * y^{n+1}\right)$ for all $x, y \in X$.

By proposition 3.4, $\tilde{\mathrm{A}}$ is an interval-valued intuitionistic fuzzy $\mathrm{n}$-fold $\mathrm{BCK}$-ideal of $\mathrm{X}$.

Conversely, It follows from (P3) and (P4) that

$$
\begin{aligned}
& \tilde{\mu}_{\mathrm{A}}\left(\left(\mathrm{x} * \mathrm{z}^{2 \mathrm{n}}\right) *\left(\mathrm{y} * \mathrm{z}^{\mathrm{n}}\right)\right)=\tilde{\mu}_{\mathrm{A}}\left(\left(\left(\mathrm{x} * \mathrm{z}^{\mathrm{n}}\right) * \mathrm{z}^{\mathrm{n}}\right) *\left(\mathrm{y} * \mathrm{z}^{\mathrm{n}}\right)\right)=\tilde{\mu}_{\mathrm{A}}\left(\left(\left(\mathrm{x} * \mathrm{z}^{\mathrm{n}}\right) *\left(\mathrm{y} * \mathrm{z}^{\mathrm{n}}\right)\right) * \mathrm{z}^{\mathrm{n}}\right) \\
& \geq \tilde{\mu}_{\mathrm{A}}\left((\mathrm{x} * \mathrm{y}) * \mathrm{z}^{\mathrm{n}}\right) \text {. } \\
& \tilde{\lambda}_{\mathrm{A}}\left(\left(\mathrm{x} * \mathrm{z}^{2 \mathrm{n}}\right) *\left(\mathrm{y} * \mathrm{z}^{\mathrm{n}}\right)\right)=\tilde{\lambda}_{\mathrm{A}}\left(\left(\left(\mathrm{x} * \mathrm{z}^{\mathrm{n}}\right) * \mathrm{z}^{\mathrm{n}}\right) *\left(\mathrm{y} * \mathrm{z}^{\mathrm{n}}\right)\right)=\tilde{\lambda}_{\mathrm{A}}\left(\left(\left(\mathrm{x} * \mathrm{z}^{\mathrm{n}}\right) *\left(\mathrm{y} * \mathrm{z}^{\mathrm{n}}\right)\right) * \mathrm{z}^{\mathrm{n}}\right) \\
& \leq \tilde{\lambda}_{A}\left((\mathrm{x} * \mathrm{y}) * \mathrm{z}^{\mathrm{n}}\right) \text {. }
\end{aligned}
$$

Using Corollary 3.5, (i-vIF2) and (i-vIF3) we get

$$
\begin{aligned}
\tilde{\mu}_{\mathrm{A}}\left(\mathrm{x} * \mathrm{z}^{\mathrm{n}}\right) \geq \tilde{\mu}_{\mathrm{A}}\left(\mathrm{x} * \mathrm{z}^{2 \mathrm{n}}\right) & \geq \min \left\{\tilde{\mu}_{\mathrm{A}}\left(\left(\mathrm{x} * \mathrm{z}^{2 \mathrm{n}}\right) *\left(\mathrm{y} * \mathrm{z}^{\mathrm{n}}\right)\right), \tilde{\mu}_{\mathrm{A}}\left(\mathrm{y} * \mathrm{z}^{\mathrm{n}}\right)\right\} \\
& \geq \min \left\{\tilde{\mu}_{\mathrm{A}}\left((\mathrm{x} * \mathrm{y}) * \mathrm{z}^{\mathrm{n}}\right), \tilde{\mu}_{\mathrm{A}}\left(\mathrm{y} * \mathrm{z}^{\mathrm{n}}\right)\right\} \\
\tilde{\lambda}_{\mathrm{A}}\left(\mathrm{x} * \mathrm{z}^{\mathrm{n}}\right) \leq \tilde{\lambda}_{\mathrm{A}}\left(\mathrm{x} * \mathrm{z}^{2 \mathrm{n}}\right) & \leq \max \left\{\tilde{\lambda}_{\mathrm{A}}\left(\left(\mathrm{x} * \mathrm{z}^{2 \mathrm{n}}\right) *\left(\mathrm{y} * \mathrm{z}^{\mathrm{n}}\right)\right), \tilde{\lambda}_{\mathrm{A}}\left(\mathrm{y} * \mathrm{z}^{\mathrm{n}}\right)\right\} \\
& \leq \max \left\{\tilde{\lambda}_{\mathrm{A}}\left((\mathrm{x} * \mathrm{y}) * \mathrm{z}^{\mathrm{n}}\right), \tilde{\lambda}_{\mathrm{A}}\left(\mathrm{y} * \mathrm{z}^{\mathrm{n}}\right)\right\} .
\end{aligned}
$$

Thus $\tilde{A}$ is an interval-valued intuitionistic fuzzy n-fold positive implicative ideal of $X$.

\section{Theorem 4.9}

Let $\tilde{\mathrm{A}}=\left(\mathrm{X}, \tilde{\mu}_{\mathrm{A}}, \tilde{\lambda}_{\mathrm{A}}\right)$ be an i-v IF set of $\mathrm{X}$, Then the following conditions are equivalent.

(i) $\tilde{A}$ is an interval-valued intuitionistic fuzzy $n$-fold positive implicative ideal of $X$.

(ii) The non-empty sets $\bigcup\left(\tilde{\mu}_{\mathrm{A}} ;\left[\mathrm{s}_{1}, \mathrm{~s}_{2}\right]\right)$ and $\mathrm{L}\left(\tilde{\boldsymbol{\lambda}}_{\mathrm{A}} ;\left[\mathrm{t}_{1}, \mathrm{t}_{2}\right]\right)$ are interval valued $\mathrm{n}$-fold positive implicative ideals of $\mathrm{X}$, for all $\left[\mathrm{s}_{1}, \mathrm{~s}_{2}\right],\left[\mathrm{t}_{1}, \mathrm{t}_{2}\right] \in \mathrm{D}[0,1]$

\section{Proof:}

The proof is straight forward.

\section{Theorem 4.10}

Let $\tilde{\mathrm{A}}=\left(\tilde{\mu}_{\mathrm{A}}, \tilde{\lambda}_{\mathrm{A}}\right)$ be an interval-valued intuitionistic fuzzy ideal of $\mathrm{X}$, then the following Conditions are equivalent:

(i) $\tilde{\mathrm{A}}=\left(\tilde{\mu}_{\mathrm{A}}, \tilde{\lambda}_{\mathrm{A}}\right)$ is an interval-valued intuitionistic fuzzy $\mathrm{n}$-fold positive implicative ideal.

(ii) $\tilde{\mathrm{A}}=\left(\tilde{\mu}_{\mathrm{A}}, \tilde{\lambda}_{\mathrm{A}}\right)$ is an interval-valued intuitionistic fuzzy $\mathrm{n}$-fold BCK-ideal of $\mathrm{X}$.

(iii) $\tilde{\mu}_{A}\left(x * y^{n}\right) \geq \tilde{\mu}_{A}\left(x * y^{n+1}\right)$ and $\tilde{\lambda}_{A}\left(x * y^{n}\right) \leq \tilde{\lambda}_{A}\left(x * y^{n+1}\right)$ for all $x, y \in X$.

(iv) $\tilde{\mu}_{\mathrm{A}}\left(\left(\mathrm{x} * \mathrm{z}^{\mathrm{n}}\right) *\left(\mathrm{y} * \mathrm{z}^{\mathrm{n}}\right)\right) \geq \tilde{\mu}_{\mathrm{A}}\left((\mathrm{x} * \mathrm{y}) * \mathrm{z}^{\mathrm{n}}\right)$ and $\tilde{\lambda}_{\mathrm{A}}\left(\left(\mathrm{x} * \mathrm{z}^{\mathrm{n}}\right) *\left(\mathrm{y} * \mathrm{z}^{\mathrm{n}}\right)\right) \leq \tilde{\lambda}_{\mathrm{A}}\left((\mathrm{x} * \mathrm{y}) * \mathrm{z}^{\mathrm{n}}\right)$

for all $\mathrm{x}, \mathrm{y}, \mathrm{z} \in \mathrm{X}$.

(v) $\mathrm{U}\left(\tilde{\mu}_{\mathrm{A}} ;\left[\mathrm{s}_{1}, \mathrm{~s}_{2}\right]\right)$ and $\mathrm{L}\left(\tilde{\lambda}_{\mathrm{A}} ;\left[\mathrm{t}_{1}, \mathrm{t}_{2}\right]\right)$ are $\mathrm{n}$-fold positive implicative ideals of $\mathrm{X}$ for all $\left[\mathrm{s}_{1}, \mathrm{~s}_{2}\right],\left[\mathrm{t}_{1}, \mathrm{t}_{2}\right] \in \mathrm{D}[0,1]$. 


\section{Proof:}

The proof is follows from the Proposition 4.7.Proposition 3.4, Theorem 4.5 and Theorem 4.9

\section{Theorem 4.11}

If $\tilde{A}=\left(\tilde{\mu}_{A}, \tilde{\lambda}_{A}\right)$ is an interval-valued intuitionistic fuzzy n-fold positive implicative ideal of $\mathrm{X}$ then

(i) for $\mathrm{x}, \mathrm{y}, \mathrm{a}, \mathrm{b} \in \mathrm{X},\left((\mathrm{x} * \mathrm{y}) * \mathrm{y}^{\mathrm{n}}\right) * \mathrm{a} \leq$ bimply $\mu_{\mathrm{A}}\left(\mathrm{x} * \mathrm{y}{ }^{\mathrm{n}}\right) \geq \min \left\{\tilde{\mu}_{\mathrm{A}}(\mathrm{a}), \tilde{\mu}_{\mathrm{A}}(\mathrm{b})\right\}$ and

$$
\tilde{\lambda}_{\mathrm{A}}\left(\mathrm{x} * \mathrm{y}^{\mathrm{n}}\right) \leq \max \left\{\tilde{\lambda}_{\mathrm{A}}(\mathrm{a}), \tilde{\lambda}_{\mathrm{A}}(\mathrm{b})\right\}
$$

(ii) for $\mathrm{x}, \mathrm{y}, \mathrm{z}, \mathrm{a}, \mathrm{b} \in \mathrm{X},\left((\mathrm{x} * \mathrm{y}) * \mathrm{z}^{\mathrm{n}}\right) * \mathrm{a} \leq$ bimply $\tilde{\mu}_{\mathrm{A}}\left(\left(\mathrm{x} * \mathrm{z}^{\mathrm{n}}\right) *\left(\mathrm{y} * \mathrm{z}^{\mathrm{n}}\right)\right) \geq \min \left\{\tilde{\mu}_{\mathrm{A}}(\mathrm{a}), \tilde{\mu}_{\mathrm{A}}(\mathrm{b})\right\}$ and $\tilde{\lambda}_{A}\left(\left(\mathrm{x} * \mathrm{z}^{\mathrm{n}}\right) *\left(\mathrm{y} * \mathrm{z}^{\mathrm{n}}\right)\right) \leq \max \left\{\tilde{\lambda}_{\mathrm{A}}(\mathrm{a}), \tilde{\lambda}_{\mathrm{A}}(\mathrm{b})\right\}$

\section{Proof:}

(i) Let $\mathrm{x}, \mathrm{y}, \mathrm{z} \in \mathrm{X}$ be such that $((\mathrm{x} * \mathrm{y}) * \mathrm{y} \mathrm{n}) * \mathrm{a} \leq \mathrm{b}$.Using Theorem 2.3,

We have $\tilde{\mu}_{\mathrm{A}}\left((\mathrm{x} * \mathrm{y}) * \mathrm{y}^{\mathrm{n}}\right) \geq \min \left\{\tilde{\mu}_{\mathrm{A}}(\mathrm{a}), \tilde{\mu}_{\mathrm{A}}(\mathrm{b})\right\}$ and $\tilde{\lambda}_{\mathrm{A}}\left((\mathrm{x} * \mathrm{y}) * \mathrm{y}{ }^{\mathrm{n}}\right) \leq \max \left\{\tilde{\lambda}_{\mathrm{A}}(\mathrm{a}), \tilde{\lambda}_{\mathrm{A}}(\mathrm{b})\right\}$.

Put $\mathrm{z}=\mathrm{y}$ in $\left(\mathrm{i}-\mathrm{vIFPI}^{\mathrm{n}}\right.$ 2) and (i-vIFPI ${ }^{\mathrm{n}}$ 3) we get

$$
\begin{aligned}
\tilde{\mu}_{\mathrm{A}}\left(\mathrm{x} * \mathrm{y}^{\mathrm{n}}\right) & \geq \min \left\{\tilde{\mu}_{\mathrm{A}}\left((\mathrm{x} * \mathrm{y}) * \mathrm{y}{ }^{\mathrm{n}}\right), \tilde{\mu}_{\mathrm{A}}\left(\mathrm{y} * \mathrm{y} \mathrm{n}^{\mathrm{n}}\right)\right\}=\min \left\{\tilde{\mu}_{\mathrm{A}}\left((\mathrm{x} * \mathrm{y}) * \mathrm{y}^{\mathrm{n}}\right), \tilde{\mu}_{\mathrm{A}}(0)\right\} \\
& =\tilde{\mu}_{\mathrm{A}}\left((\mathrm{x} * \mathrm{y}) * \mathrm{y}^{\mathrm{n}}\right) \geq \min \left\{\tilde{\mu}_{\mathrm{A}}(\mathrm{a}), \tilde{\mu}_{\mathrm{A}}(\mathrm{b})\right\} \text { and } \\
\tilde{\lambda}_{\mathrm{A}}\left(\mathrm{x} * \mathrm{y}^{\mathrm{n}}\right) & \leq \max \left\{\tilde{\lambda}_{\mathrm{A}}\left((\mathrm{x} * \mathrm{y}) * \mathrm{y}^{\mathrm{n}}\right), \tilde{\lambda}_{\mathrm{A}}\left(\mathrm{y} * \mathrm{y}^{\mathrm{n}}\right)\right\}=\max \left\{\tilde{\lambda}_{\mathrm{A}}\left((\mathrm{x} * \mathrm{y}) * \mathrm{y}^{\mathrm{n}}\right), \tilde{\lambda}_{\mathrm{A}}(0)\right\} \\
& =\tilde{\lambda}_{\mathrm{A}}\left((\mathrm{x} * \mathrm{y}) * \mathrm{y}^{\mathrm{n}}\right) \leq \max \left\{\tilde{\lambda}_{\mathrm{A}}(\mathrm{a}), \tilde{\lambda}_{\mathrm{A}}(\mathrm{b})\right\} .
\end{aligned}
$$

Therefore, $\tilde{\mu}_{A}\left(x * y^{n}\right) \geq \min \left\{\tilde{\mu}_{A}(a), \tilde{\mu}_{A}(b)\right\}$ and $\tilde{\lambda}_{A}\left(x * y^{n}\right) \leq \max \left\{\tilde{\lambda}_{A}(a), \tilde{\lambda}_{A}(b)\right\}$

(ii) Let $x, y, z \in X$ be such that $\left((x * y) * z{ }^{n}\right) * a \leq b$. It follows from Theorems 4.5, Theorems 4.6 and By (i).

We obtain $\tilde{\mu}_{\mathrm{A}}\left(\left(\mathrm{x} * \mathrm{z}^{\mathrm{n}}\right) *\left(\mathrm{y} * \mathrm{z}^{\mathrm{n}}\right)\right) \geq \tilde{\mu}_{\mathrm{A}}\left((\mathrm{x} * \mathrm{y}) * \mathrm{z}^{\mathrm{n}}\right) \geq \min \left\{\tilde{\mu}_{\mathrm{A}}(\mathrm{a}), \tilde{\mu}_{\mathrm{A}}(\mathrm{b})\right\}$ and

$\tilde{\lambda}_{\mathrm{A}}\left(\left(\mathrm{x} * \mathrm{z}^{\mathrm{n}}\right) *\left(\mathrm{y} * \mathrm{z}^{\mathrm{n}}\right)\right) \leq \tilde{\lambda}_{\mathrm{A}}\left((\mathrm{x} * \mathrm{y}) * \mathrm{z}^{\mathrm{n}}\right) \leq \max \left\{\tilde{\lambda}_{\mathrm{A}}(\mathrm{a}), \tilde{\lambda}_{\mathrm{A}}(\mathrm{b})\right\}$

This completes the proof.

\section{Theorem 4.16.}

(Extension property for an interval-valued intuitionistic fuzzy n-fold positive implicative ideal). Let $\tilde{\mathrm{A}}=\left(\tilde{\mu}_{\mathrm{A}}, \tilde{\lambda}_{\mathrm{A}}\right)$ and $\tilde{\mathrm{B}}=\left(\tilde{\mu}_{\mathrm{B}}, \tilde{\lambda}_{\mathrm{B}}\right)$ be an interval-valued intuitionistic fuzzy ideals of $\mathrm{X}$ such that $\tilde{\mathrm{A}}(0)=\tilde{\mathrm{B}}(0)$ and $\tilde{\mathrm{A}} \subseteq \tilde{\mathrm{B}}$, that is, $\tilde{\mu}_{\mathrm{A}}(0)=\tilde{\mu}_{\mathrm{B}}(0), \tilde{\lambda}_{\mathrm{A}}(0)=\tilde{\lambda}_{\mathrm{B}}(0)$ and $\tilde{\mu}_{\mathrm{A}}(\mathrm{x}) \leq \tilde{\mu}_{\mathrm{B}}(\mathrm{x}), \tilde{\lambda}_{\mathrm{A}}(\mathrm{x}) \geq \tilde{\lambda}_{\mathrm{B}}(\mathrm{x})$, for all $\mathrm{x} \in \mathrm{X}$. If $\tilde{\mathrm{A}}=\left(\tilde{\mu}_{\mathrm{A}}, \tilde{\lambda}_{\mathrm{A}}\right)$ is an interval-valued intuitionistic fuzzy $\mathrm{n}$-fold positive implicative ideal of $\mathrm{X}$ then so is $\tilde{\mathrm{B}}$.

\section{Proof:}

It is sufficient to show that $B=\left(X, \mu_{B}, \lambda_{B}\right)$ satieties the inequalities $\tilde{\mu}_{B}\left(x * y{ }^{n}\right) \geq \tilde{\mu}_{B}(x * y=n+1)$ and $\tilde{\lambda}_{B}\left(\mathrm{x} * \mathrm{y}^{\mathrm{n}}\right) \leq \tilde{\lambda}_{\mathrm{B}}\left(\mathrm{x} * \mathrm{y}^{\mathrm{n}+1}\right)$ for all $\mathrm{x}, \mathrm{y} \in \mathrm{X}$. 
Let $\mathrm{x}, \mathrm{y} \in \mathrm{X}$.Using (BCK-3), (P3) and Proposition 3.4, we get

$$
\begin{aligned}
& \tilde{\mu}_{B}(0)=\tilde{\mu}_{A}(0)=\tilde{\mu}_{A}\left(\left(x *\left(x * y^{n+1}\right)\right) * y^{n+1}\right) \leq \tilde{\mu}_{A}\left(\left(x *\left(x * y^{n+1}\right)\right) * y^{n}\right) \\
& =\tilde{\mu}_{A}\left(\left(\mathrm{x} * \mathrm{y}^{\mathrm{n}}\right) *\left(\mathrm{x} * \mathrm{y}^{\mathrm{n}+1}\right)\right) \\
& \leq \tilde{\mu}_{\mathrm{B}}\left(\left(\mathrm{x} * \mathrm{y}^{\mathrm{n}}\right) *\left(\mathrm{x} * \mathrm{y}^{\mathrm{n}+1}\right) \text { and } \tilde{\lambda}_{\mathrm{B}}(0)=\tilde{\lambda}_{\mathrm{A}}(0)=\tilde{\lambda}_{A}\left(\left(\mathrm{x} *\left(\mathrm{x} * \mathrm{y}^{\mathrm{n}+1}\right)\right) * \mathrm{y}^{\mathrm{n}+1}\right)\right. \\
& \geq \tilde{\lambda}_{A}\left(\left(\mathrm{x} *\left(\mathrm{x} * \mathrm{y}^{\mathrm{n}+1}\right)\right) * \mathrm{y}^{\mathrm{n}}\right) \\
& =\tilde{\lambda}_{A}\left(\left(\mathrm{x} * \mathrm{y}^{\mathrm{n}}\right) *\left(\mathrm{x} * \mathrm{y}^{\mathrm{n}+1}\right)\right) \text {. } \\
& \geq \tilde{\lambda}_{B}\left(\left(\mathrm{x} * \mathrm{y}^{\mathrm{n}}\right) *\left(\mathrm{x} * \mathrm{y}^{\mathrm{n}+1}\right)\right. \text {. }
\end{aligned}
$$

Therefore, $\tilde{\mu}_{\mathrm{B}}(0) \leq \tilde{\mu}_{\mathrm{B}}\left(\left(\mathrm{x} * \mathrm{y}^{\mathrm{n}}\right) *\left(\mathrm{x} * \mathrm{y}^{\mathrm{n}+1}\right)\right.$ and $\tilde{\lambda}_{\mathrm{B}}(0) \geq \tilde{\lambda}_{\mathrm{B}}\left(\left(\mathrm{x} * \mathrm{y}^{\mathrm{n}}\right) *\left(\mathrm{x} * \mathrm{y}^{\mathrm{n}+1}\right)\right.$ for all $\mathrm{x}, \mathrm{y} \in \mathrm{X}$. It follows from (i-vIF1), (i-vIF2) and (i-vIF3) that

$$
\begin{aligned}
\tilde{\mu}_{\mathrm{B}}\left(\mathrm{x} * y^{\mathrm{n}}\right) & \geq \min \left\{\tilde{\mu}_{\mathrm{B}}\left(\left(\mathrm{x} * y^{\mathrm{n}}\right) *(\mathrm{x} * \mathrm{y}+1)\right), \tilde{\mu}_{\mathrm{B}}(\mathrm{x} * \mathrm{y}+1)\right\} \\
& \geq \min \left\{\tilde{\mu}_{\mathrm{B}}(0), \tilde{\mu}_{\mathrm{B}}\left(\mathrm{x} * \mathrm{y}^{\mathrm{n}+1}\right)\right\}=\tilde{\mu}_{\mathrm{B}}\left(\mathrm{x} * \mathrm{y}^{\mathrm{n}+1}\right)
\end{aligned}
$$

And $\quad \tilde{\lambda}_{\mathrm{B}}\left(\mathrm{x} * \mathrm{y}^{\mathrm{n}}\right) \leq \max \left\{\tilde{\lambda}_{\mathrm{B}}\left(\left(\mathrm{x} * \mathrm{y}^{\mathrm{n}}\right) *\left(\mathrm{x} * \mathrm{y}^{\mathrm{n}+1}\right)\right), \tilde{\lambda}_{\mathrm{B}}\left(\mathrm{x} * \mathrm{y}^{\mathrm{n}+1}\right)\right\}$

$$
\leq \max \left\{\tilde{\lambda}_{\mathrm{B}}(0), \lambda_{\mathrm{B}}\left(\mathrm{x} * \mathrm{y}^{\mathrm{n}+1}\right)\right\}=\tilde{\lambda}_{\mathrm{B}}\left(\mathrm{x} * \mathrm{y}^{\mathrm{n}+1}\right) .
$$

Therefore, $\tilde{\mu}_{\mathrm{B}}\left(\mathrm{x} * y^{\mathrm{n}}\right) \geq \tilde{\mu}_{\mathrm{B}}\left(\mathrm{x} * \mathrm{y}^{\mathrm{n}+1}\right)$ and $\tilde{\lambda}_{\mathrm{B}}\left(\mathrm{x} * \mathrm{y}^{\mathrm{n}}\right) \leq \tilde{\lambda}_{\mathrm{B}}\left(\mathrm{x} * \mathrm{y}^{\mathrm{n}+1}\right)$ for all $\mathrm{x}, \mathrm{y} \in \mathrm{X}$.

Thus $\tilde{B}=\left(\tilde{\mu}_{B}, \tilde{\lambda}_{B}\right)$ is an interval-valued intuitionistic fuzzy n-fold positive implicative ideal of $X$.

\section{Conclusion :}

Every interval-valued intuitionistic fuzzy n-fold BCK- ideal and interval-valued intuitionistic fuzzy $n$-fold positive implicatice BCK- ideal of $X$ is an interval-valued intuitionistic fuzzy deal of $X$. An interval-valued intuitionistic fuzzy $n$-fold positive implicative ideal of $X$ satisfies the inequalities

$$
\begin{aligned}
& \tilde{\mu}_{A}\left(\left(\mathrm{x} * \mathrm{z}^{\mathrm{n}}\right) *\left(\mathrm{y} * \mathrm{z}^{\mathrm{n}}\right)\right) \geq \tilde{\mu}_{A}\left((\mathrm{x} * \mathrm{y}) * \mathrm{z}^{\mathrm{n}}\right) \text { and } \\
& \tilde{\lambda}_{A}\left(\left(\mathrm{x} * \mathrm{z}^{\mathrm{n}}\right) *\left(\mathrm{y} * \mathrm{z}^{\mathrm{n}}\right)\right) \leq \tilde{\lambda}_{A}\left((\mathrm{x} * \mathrm{y}) * \mathrm{z}^{\mathrm{n}}\right) \text { for all } \mathrm{x}, \mathrm{y}, \mathrm{z} \in \mathrm{X} .
\end{aligned}
$$

Interval-valued intuitionistic fuzzy $n$-fold positive implicative ideal of $X$ if and only if it is an intuitionistic fuzzy n-fold BCK-ideal of X. Characterizations of these notions and extension property of interval-valued intuitionistic fuzzy n-fold positive implicative ideals are proved. 


\section{References :}

[1] K. T. Atanassov, Intuitionistic fuzzy sets, Fuzzy Sets and Systems 20, 87-96 (1986).

[2] K. T Atanassov, and G. Gargov, Interval valued intuitionistic fuzzy sets, Fuzzy sets and Systems, 31(1989), 343-349.

[3] K.T. Atanassov, Operators over interval valued intuitionistic fuzzy sets, Fuzzy Sets and Systems 64 (1994) 159-174.

[4] M. Akram, K.H Dar, and B.L. Meng , K.P.Shum, Interval-valued intuitionistic fuzzy ideals of K-algebras, WSEAS Trans. Math. 7 (9) (2008) 559-568.

[5] R. Biswas, Rosenfeld's fuzzy subgroups with interval valued membership function, Fuzzy Sets and Systems 1 (63) (1994), 87-90.

[6] Y. Hung, and Z.Chen, On ideals in BCK-algebras, Math. Japan. 50(1999), no.2,211226.CMP 1718 851. Zb1 938.06018.

[7] Y. Imai, and K.Iseki, on axioms systems of proposition calculi XIV, Proc. Japan Acad, (1966) 19-22.

[8] Y.B. Jun, and E.H. Roh , Fuzzy commutative ideals of BCK-algebras, Fuzzy sets and Systems, 64 (1994) 401-405.

[9] Y.B Jun, and K.H. Kim, On foldness of fuzzy positive implicative ideals of BCKalgebras, Hindwi Publications, 24 (9), 525-537(2001).

[10] Y.B. Jun, On foldness of fuzzy implicative / Commutative ideals of BCK-algebras, Hindawi publishing corp, 27(7) (2001), 419-424.

[11] J. Meng , Y.B. Jun, and H.S. Kim , Fuzzy implicative ideals of BCK-algebras, Fuzzy Sets and Systems, 89(2) (1997), 243-248.

[12] S.M. Mostafa , Fuzzy implicative ideals in BCK-algebra, Fuzzy sets and systems, 87 (1997), 361-368.

[13] B. Satyanarayana and R. Durga Prasad, on foldness of intuitionistic fuzzy implicative and commutative ideals of BCK-algebras, Aryabatta. J. of .math.\& information 3(1),(2011),139- 150.

[14] B . Satyanarayana and R. Durga Prasad, on foldness of intuitionistic fuzzy positive implicative ideals of BCK-algebras, Research J. Pure algebra,1(2),(2011),40-51.

[15] L. A. Zadeh, Fuzzy sets, Inform. Control 8, 338-353(1965). 